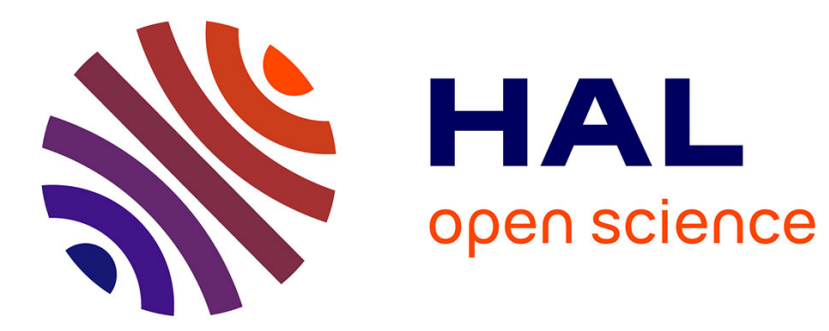

\title{
Experimental demonstration of directive pulsed wavefront generation in reverberation chambers
}

Houmam Moussa, Andrea Cozza, Michel Cauterman

\section{To cite this version:}

Houmam Moussa, Andrea Cozza, Michel Cauterman. Experimental demonstration of directive pulsed wavefront generation in reverberation chambers. Electronics Letters, 2010, 46 (9), pp.623-624. hal00505990

\section{HAL Id: hal-00505990 \\ https://hal.science/hal-00505990}

Submitted on 25 Nov 2010

HAL is a multi-disciplinary open access archive for the deposit and dissemination of scientific research documents, whether they are published or not. The documents may come from teaching and research institutions in France or abroad, or from public or private research centers.
L'archive ouverte pluridisciplinaire HAL, est destinée au dépôt et à la diffusion de documents scientifiques de niveau recherche, publiés ou non, émanant des établissements d'enseignement et de recherche français ou étrangers, des laboratoires publics ou privés. 


\title{
Experimental demonstration of directive and polarized pulsed fields generation within a
} reverberation chamber

\author{
Houmam Moussa, Andrea Cozza and Michel Cauterman
}

In this paper, the first experimental demonstration of coherent directive and polarized wavefront generation within a reverberation chamber is presented. This feature is possible with the use of a time-reversal electromagnetic chamber, a concept that we previously introduced which mainly relies on the use of time-reversal techniques in a highly reverberating medium. The rationale for developing such a system is to provide the control of a field distribution in time and space without any mechanical move of neither sources nor an equipment under test while preserving the high-intensity field levels achieved with reverberation chambers.

\section{INTRODUCTION}

We have recently shown, [1], [2], that the use of time-reversal techniques, as applied in highly reverberating mediums, allows to control the spatial distribution and polarization of pulsed wavefronts as long as the system developed is reciprocal and time invariant. The interest of the system proposed, called a time reversal electromagnetic chamber (TREC), resides in the ability to control the wavefront propagation direction, polarization, and time dependence in real-time. This is achieved by modifying the excitation signal applied to a set of antennas, placed within the facility. These antennas are referred to as the timereversal mirror (TRM) in the literature [3]. The advantage here is that there is no need of any mechanical movements of the antennas or the equipment under test (EUT) thanks to the multiple reflections on the cavity walls. The procedure for obtaining these signals will be described in the next Section.

In this letter, we prove that the TREC can be implemented in a real life-scenario thanks to an experimental validation of the system. 


\section{COHERENT FIELD GENERATION}

A TREC is composed of an arbitrary shaped cavity with a set of $M$ antennas, used as TRM, and usually located near its walls. Each one of these is fed by a signal $y_{i}(t)$, with $i$ the index of one TRM antenna, in order to generate a wavefront converging towards the EUT. We have shown in [1] that the procedure to obtain these signals requires the definition of a surface $\Gamma$, over which the field generated by the TRM antennas is sampled over $N$ different locations. Thus, we can determine the transfer functions $\phi_{i j}^{p}(\omega)$ between the i-th port of the TRM antenna and the E-field measured at different positions over $\Gamma$. In our notation, the index $j$ stands for one position over $\Gamma$ and $p$ a given field polarization. The index $p$ takes the values $\{1,2\}$ for the two field components tangential to $\Gamma$, i.e., $E_{\theta}$ and $E_{\phi}$. The surface $\Gamma$ needs to be positioned between the wavefront direction of propagation and the region where an EUT can be placed, as the wavefront is going to pass through $\Gamma$ in order to impinge on the EUT. With the analyses carried out in [1], we have also demonstrated that, as long as the system is linear and reciprocal, we can synthesize the signals $y_{i}(t)$ that have to be applied on each TRM antenna port. They are given by computing the inverse Fourier transform of

$$
Y_{i}(\omega)=X(\omega) \sum_{p=1}^{2} \sum_{j=1}^{N} \alpha_{j}^{p *} \phi_{i j}^{p *}(\omega)
$$

where $X(\omega)$ is the spectrum of the user-defined signal setting the time dependence of the wavefront, e.g., a gaussian pulse. The mathematical operator * stands for the phase conjugate. The $\alpha_{j}^{p}$ are weights associated to the locations of the $N$ field sampling points, along each polarization $p$. These weights enable the synthesis of the signals injected on the TRM antennas in order to obtain a converging wavefront towards $\Gamma$. This wavefront closely approximates the diverging wavefront that would have been radiated in free space by a phased-array of elementary electric dipoles distributed over $\Gamma$ with excitations $\left\{\alpha_{j}^{p}\right\}$ applied to them. This implies that the field distribution and its polarization can be modified by changing the $\left\{\alpha_{j}^{p}\right\}$ and computing (1) in order to synthesize the required TRM signals in real time. Indeed, the only unknown quantities in (1) are the $\left\{\phi_{i j}^{p}\right\}$ : they can be determined in a number of ways, e.g., by means of vector network analyzer connected between the antenna ports and an E-field probe.

We desired to validate experimentally these ideas. In order to make this demonstration easier, we studied the system performance with a transfer-function approach. The main advantage of this approach is that the E-field propagating towards the EUT inside the TREC can be computed for many set of weights $\alpha_{j}^{p}$ with no need of repeating the measurements. 
We are interested in observing the spatial distribution and the time evolution of the E-field propagating inside the TREC. To this end, we need to know the transfer functions $\left\{\psi_{i k}^{q}\right\}$ between the port of the TRM antennas and the spatial location where the field is going to be observed. The polarization index $q$ is related to the cartesian components of the field with $q=\{1,2,3\}$ for respectively, $E_{x}, E_{y}$ and $E_{z}$ while $k$ is the index of the probe position on a planar test surface $\Upsilon$. The E-field $E_{k}^{q}(\omega)$, at a location $k$, can be computed by means of :

$$
E_{k}^{q}(\omega)=\sum_{q=1}^{3} \sum_{i=1}^{M} Y_{i}(\omega) \psi_{i k}^{q}(\omega)
$$

The test surface $\Upsilon$ is composed of three parts corresponding to the different positions of a planar styrofoam support placed asides $\Gamma$ as shown in Fig. 2.

\section{EXPERIMENTAL RESULTS}

In order to demonstrate the properties of the TREC in a real-life scenario, measurements were carried out in Supélec's RC, characterized by physical dimensions $3.08 \times 1.84 \times 2.44 \mathrm{~m}^{3}$ along, respectively, the $\mathrm{x}, \mathrm{y}$ and $\mathrm{z}$ axis. The setup was composed of twenty bow-tie printedcircuit antennas distributed over the four cavity walls at different heights, their axes oriented normally to the walls, used as TRM antennas. The antenna cables were pinned to the walls and linked to a multiplexer shielded by a metallic box. A vector network analyzer was connected between the output of the multiplexer and the port of an E-field probe. The probe was of an all-optical linearly polarized model EFS-105, mounted on two different styrofoam supports: the first one was in the shape of an arch, ensuring the measurements of the $\left\{\phi_{i j}^{p}\right\}$. The arch was rotated with a complete revolution around its vertical axis in order to describe $\Gamma$ as an hemisphere. The second support was a parallelepiped designed to measure the $\left\{\psi_{i k}^{q}\right\}$ over a cartesian grid, i.e., the surface $\Upsilon$ composed of three elements as shown Fig. 2 and used as a planar monitor in order to observe the wavefront propagation towards the arch. The distance between two samples was $\lambda / 4$, with $\lambda$ the wavelength associated to the maximum frequency of $X(\omega)$ in (1). The type of signal used for $X(\omega)$ was a gaussian pulse with a central frequency of $850 \mathrm{MHz}$ a $3 \mathrm{~dB}$ bandwidth of $115 \mathrm{MHz}$. During all the measurements, the stirrer was set on a fixed position and was not used, acting as a static part of the cavity.

We were interested in the cross-polarization rejection and the spatial distribution of the field for two cuts of the hemisphere $\Gamma$; these correspond to a position of the arch along respectively the $x$ and $y$ axis in the chamber. In order to excite only one of the two tangential polarizations at a time, either $E_{\theta}$ or $E_{\phi}$, we have set to zero the weights $\alpha_{j}^{p}$ associated to the non-excited 
polarization in (1). Meanwhile, the weights of the excited polarization were all set to the same value in order to generate a converging hemispherical wavefront in the plane of the arch, i.e., we excited only one meridian of $\Gamma$. Then we observed the field distribution on the two arch positions, occurring at the instant when the peak field is obtained, for both polarizations. These amplitudes were normalized in order to have a $0 \mathrm{~dB}$ maximum gain. Fig. 1 proves that the cross-polarization rejections are higher than $25 \mathrm{~dB}$ with a mean value of about $30 \mathrm{~dB}$. These rejection levels are in a good agreement with results shown in [4]. We can also remark here that the TREC system manages to preserve the same profile of the peak field distribution over the arch for each position studied, even with the presence of elements breaking the symmetry of the configuration : TRM antennas at different positions, a stirrer. This, shows that the system proposed is able to generate a wavefront independent of the actual propagation channel; this implies that it could be a reliable tool for coherent and polarized wavefronts generation.

The results in Fig. 2 show the time evolution of the field distribution on the planar test surfaces $\Upsilon$.The results were obtained by taking the norm of the field from the three cartesian components on $\Upsilon$, when placed on both sides of the arch and inside it. The weights $\left\{\alpha_{j}^{p}\right\}$ were selected in order to excite only the polarization $E_{\theta}$ and generate two different wavefronts : one describes a propagation coming only from the whole upper-half of the arch, and the other one from the its upper-right. We can clearly see the propagation of a wavefront converging towards the arch center in both cases, as the time increases with a step of $0.5 \mathrm{~ns}$. We can also observe that we have a good signal-to-noise ratio at the instant of the peak field as the energy ratio between the coherent field and the incoherent background field average level is around $20 \mathrm{~dB}$. Also, we have verified that the field amplitudes increase proportionally to $1 / r$ from the TRM antennas to the arch center, which correspond to the radial dependence of a spherical wavefront propagating in free space conditions.

\section{CONCLUSIONS}

We have proven experimentally that the TREC is an interesting testing tool as it allows, with a high flexibility, to generate and control the polarization, the spatial distribution and the time dependency of coherent fields within RCs. These features are enabled without any mechanical movement of either the sources or the EUT, conversely to what can be achieved with anechoic chambers, by operating on the signal applied to the excitation antennas. 


\section{REFERENCES}

[1] H. Moussa, A. Cozza, M. Cauterman, "Directive wavefronts inside a Time Reversal Electromagnetic Chamber," Proc. IEEE Int. Symp. on Electromagnetic Compatibility, Austin, Texas, August, 2009.

[2] A. Cozza, H. Moussa, "Enforcing a deterministic polarization in a reverberating environment," Electronic Letters, vol. 45, no. 25, pp. 1299-1301, Dec. 2009.

[3] M. Fink, "Time reversal of ultrasonic fields: part I. Basic principles," IEEE Trans. Ultrason. Ferro-electr. Freq. Control, vol. 39, no. 5, pp. 555-566, Sept. 1992.

[4] A. Cozza, H. Moussa, "Generating Coherent Polarized Pulsed Fields in a Reverbaration Chamber," accepteted to 2010 Asia-Pacific Symposium on Electromagnetic Compatibility, Beijing, China, April, 2010.

\section{AUTHORS AFFILIATION}

Houmam Moussa, e-mail : houmam.moussa@lss.supelec.fr (Département de Recherche en Électromagnétisme, SUPELEC, 3 Rue Joliot-Curie, 91192 Gif sur Yvette).

Andrea Cozza, e-mail : andrea.cozza@supelec.fr (Département de Recherche en Électromagnétisme, SUPELEC, 3 Rue Joliot-Curie, 91192 Gif sur Yvette).

Michel Cauterman, e-mail : michel.cautermanelss.supelec.fr (Département de Recherche en Électromagnétisme, SUPELEC, 3 Rue Joliot-Curie, 91192 Gif sur Yvette). 


\section{FIGURE CAPTIONS}

Fig. 1: Distribution of the wavefront evaluated for two cuts of $\Gamma$ : (a) the excited polarization is $E_{\theta}$; (b) the excited polarization is $E_{\phi}$. The indexes $x$ and $y$ in the legends stand respectively for a position of the arch along the $x$ - and the $y$-axis of the cavity.

Fig. 2 : Time evolution of the field distribution in a cut along the cavity x-axis: (a) upper-half hemispherical wavefront converging towards $\Gamma$; (b) right-half wavefront converging towards $\Gamma$. The time step is $0.5 \mathrm{~ns}$. The black curve represents the surface $\Gamma$ cut along the $x$-axis. 
Figure 1.

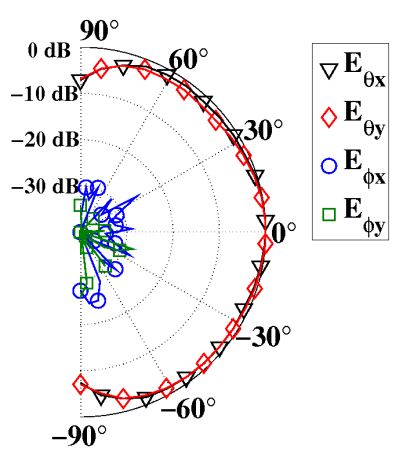

(a)

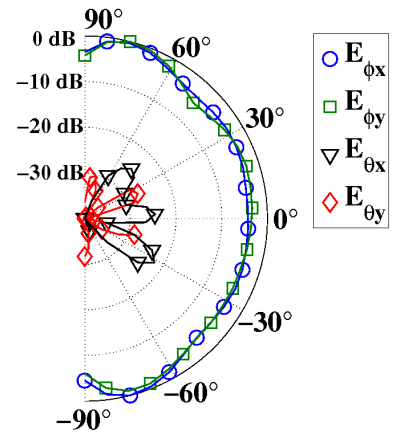

(b) 
Figure 2.

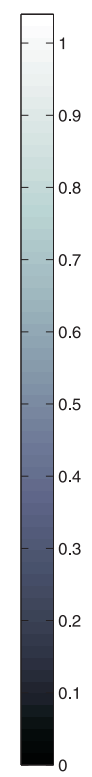

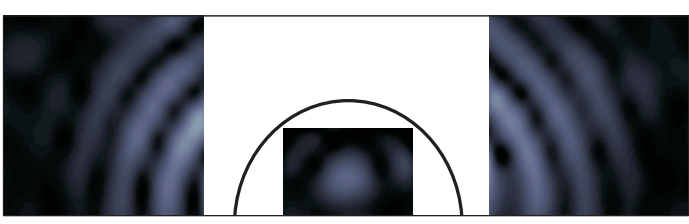
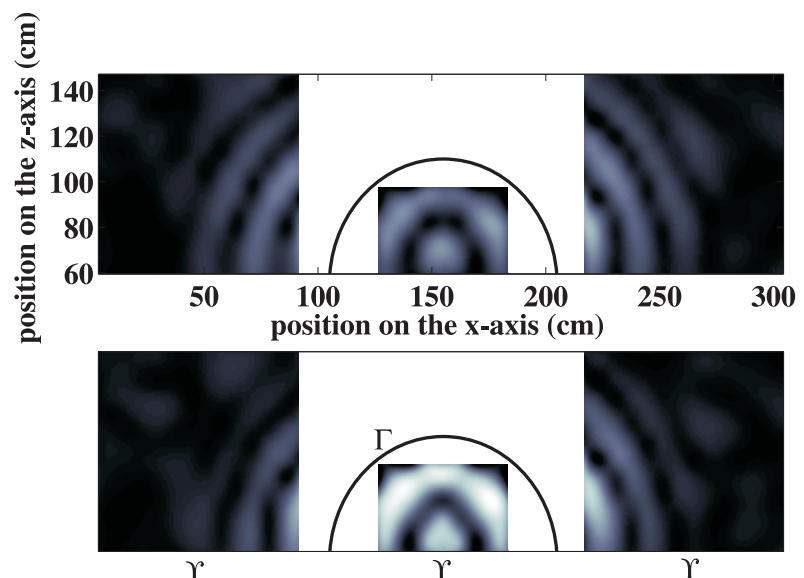

(a)

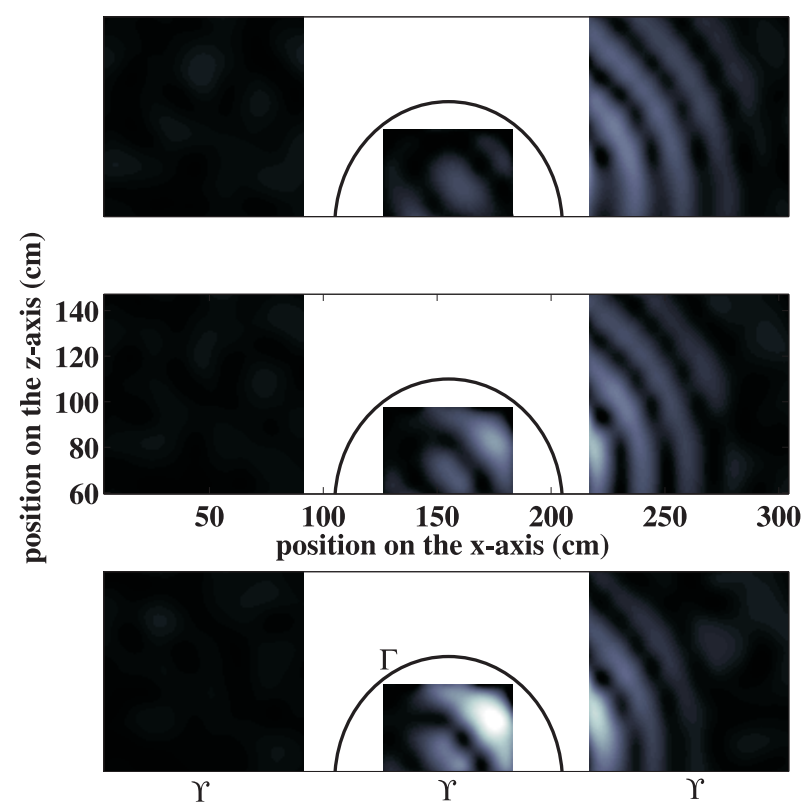

(b) 